\title{
Rigorous derivation of coherent resonant tunneling time and velocity in finite periodic systems
}

\author{
C. Pacher, W. Boxleitner and E. Gornik \\ Institut für Festkörperelektronik, Technische Universität Wien, A-1040 Wien, Austria and \\ ARC Seibersdorf Research GmbH, Donau-City Straße 1/4, A-1220 Wien, Austria
}

(Dated: February 9, 2020)

\begin{abstract}
The velocity $v_{\text {res }}$ of resonant tunneling electrons in finite periodic structures is analytically calculated in two ways. The first method is based on the fact that a transmission of unity leads to a coincidence of all still competing tunneling time definitions. Thus, having an indisputable resonant tunneling time $\tau_{\text {res }}$, we apply the natural definition $v_{\text {res }}=L / \tau_{\text {res }}$ to calculate the velocity. For the second method we combine Bloch's theorem with the transfer matrix approach to decompose the wave function into two Bloch waves. Then the expectation value of the velocity is calculated. Both different approaches lead to the same result, showing their physical equivalence. The obtained resonant tunneling velocity $v_{\text {res }}$ is smaller or equal to the group velocity times the magnitude of the complex transmission amplitude of the unit cell. Only at energies where the unit cell of the periodic structure has a transmission of unity $v_{\text {res }}$ equals the group velocity. Numerical calculations for a GaAs/AlGaAs superlattice are performed. For typical parameters the resonant velocity is below one third of the group velocity.
\end{abstract}

PACS numbers: 03.65.Xp,73.21.Cd,02.60.Lj

Keywords: tunneling time, resonant tunneling, periodic, Bloch, group velocity

\section{INTRODUCTION}

There has been an ongoing debate about the time an electron spends when it passes through a classically forbidden region (e.g. a rectangular barrier) for many decades. Despite a number of review articles ${ }^{1,2}$ and many papers up to now there still exist different definitions of tunneling time. Most of the studies have been performed in one of the following frameworks: (i) wave packet analysis $1,3,4,5,6$, (ii) dynamic paths including Feynman paths 7.8.9, (iii) physical clocks 10.11 .12 .13 .14 .15 .16 .17 , (iv) flux-flux correlation functions ${ }^{18}$, (v) theory of weak measurements ${ }^{19}$, and (vi) combinations of the former ${ }^{20}$.

The real phase or delay time

$$
\tau_{\text {phase }}(E)=\frac{\partial \arg t(E)}{\partial \omega}=\hbar \frac{\partial \arg t(E)}{\partial E},
$$

where $t(E)$ is the complex transmission coefficient as a function of energy, and $\omega$ is the angular frequency, arises from a stationary-phase argument for the transmitted wave packet. Many of the approaches (ii) to (vi) result at first in complex transmission tunneling times, given by one of the following expressions:

$$
\begin{aligned}
\tau_{T}^{E}(E) & =-i \hbar \frac{\partial \ln t(E)}{\partial E}=\tau_{\text {phase }}(E)-i \hbar \frac{\partial \ln |t(E)|}{\partial E} \\
\tau_{T}^{V}(E) & =i \hbar \frac{\partial \ln t(E)}{\partial V} \\
\tau_{T}^{\delta V}(E) & =i \hbar \frac{\delta \ln t(E)}{\delta V(x)}=\tau_{T}^{E}(E)-i \hbar \frac{r(E)+r^{\prime}(E)}{4 E}
\end{aligned}
$$

where $\delta / \delta V(x)$ denotes the functional derivative with respect to the potential $V(x)$, and $r(E)$ and $r^{\prime}(E)$ are the reflection amplitudes for particles coming from the left and right side, respectively. The corresponding reflection times $\tau_{R}^{X}(E)$ are given by the substitution of $t(E)$ by $r(E)$ in the left equalities in Eqs. (2) to (4). In the framework of physical clocks 10.11 .12 .13 .14 .15 .16 .17 the resulting times have been the real or imaginary part or the absolute value of one of the times in (2) and (3).

On the other hand, it was shown that the simultaneous process of (a) the determination whether a particle is transmitted and (b) if so, how long it took to traverse the barrier, corresponds to two non-commuting observables 21 . There was also some direct evidence that the imaginary part of the tunneling time results from the backaction on the particle due to the measurement process 19 .

In contrast, the time an electron spends under the barrier, either finally reflected by or transmitted through the barrier, is consistently given by the dwell time ${ }^{22}$, also called sojourn time, which is defined as the ratio of the number of particles within the barrier (extending from $a$ to $b)$ to the incident flux ${ }^{11}$ :

$$
\tau_{D}(E)=\frac{1}{v_{\text {in }}} \int_{a}^{b}|\Psi|^{2} d x .
$$

A Hermitian sojourn time operator exists ${ }^{23}$, which shows that this time is measurable.

Again there has been no general agreement whether a relation of the following form must hold:

$$
\tau_{D}(E)=|t(E)|^{2} \tau_{T}(E)+|r(E)|^{2} \tau_{R}(E) .
$$

Based on the argument that reflection and transmission are mutually exclusive events, that exhaust all possibilities in the sense of Feynman, this relation served as a point of focus in an early review ${ }^{1}$. The complex tunneling times $\tau_{T, R}^{\delta V}$ fulfill Eq. (6) ? $\frac{7}{?}$ Nevertheless, the arguments for 
Eq. (6) have also been critisized arguing that the used approach goes beyond Feynman's original interpretation ${ }^{2}$.

\section{Tunneling times in case of transmission equals unity}

Given that $|t|=1$, for a certain energy, it was shown that phase time and dwell time are identical, not only for the single barrier (at energies higher than the potential energy of the barrier) $\stackrel{11}{\underline{1}}$, but also for arbitrary structures 1.5. The tunneling times $\tau_{T}^{E}, \tau_{T}^{V}$ and $\tau_{T}^{\delta V}$, Eqs. (2) to (44), also simplify to the phase time $\tau_{\text {phase }}$, Eq. (11), for $|t|=1$ in any arbitrary structure. Thus we have

$$
\begin{aligned}
\left|t\left(E^{\prime}\right)\right|=1 & \Rightarrow \\
\tau_{\text {phase }}\left(E^{\prime}\right) & =\tau_{D}\left(E^{\prime}\right)=\tau_{T}^{E}\left(E^{\prime}\right)=\tau_{T}^{V}\left(E^{\prime}\right)=\tau_{T}^{\delta V}\left(E^{\prime}\right) .
\end{aligned}
$$

In accordance with the real character of the phase time the aforementioned problem of non-commuting observables vanishes in the case $|t(E)|=1$, since all particles tunnel finally through the structure; there is neither reflection nor interference between reflected and transmitted particles.

Stimulated by comments in Ref. 2 and recent results from the theory of finite periodic systems ${ }^{24.25}$, here we study the tunneling time of electrons that tunnel resonantly through finite periodic systems. In contrast to tunneling through single barriers, periodic systems have a transmission probability of unity below the barrier potential at the individual transmission resonances, which form allowed energy bands. Due to Eq. (7) we can choose any time definition, Eqs. (11) to (5), to calculate the tunneling time at resonance. We will use the phase delay time, Eq. (11), and make use of the results obtained in Refs. 25 and 26 recently.

At first view, a similiar approach to the phase time is the concept of the group velocity

$$
v_{g}=\frac{\partial \omega}{\partial k}
$$

which is the velocity of the envelope of a propagating wave packet in a medium. Here $\omega$ is the angular frequency and $k$ is the wave number. The function $\omega(k)$ is normally referred to as dispersion. The solutions of the Schrödinger equation for a periodic potential also yield a (band) dispersion relation between the Bloch wave number $q$ and the angular frequency $\omega$. Using $E=\hbar \omega$, the group velocity then reads:

$$
v_{g}=\left(\hbar \frac{\partial q}{\partial E}\right)^{-1} .
$$

In Ref. 2 the following relation between the tunneling time and the group velocity was given (for the tight binding limit), neglecting terms due to the matching of the wave functions at the ends of the system:

$$
v_{g} \cong L /\left|\tau_{T}^{E}\right| \text {. }
$$

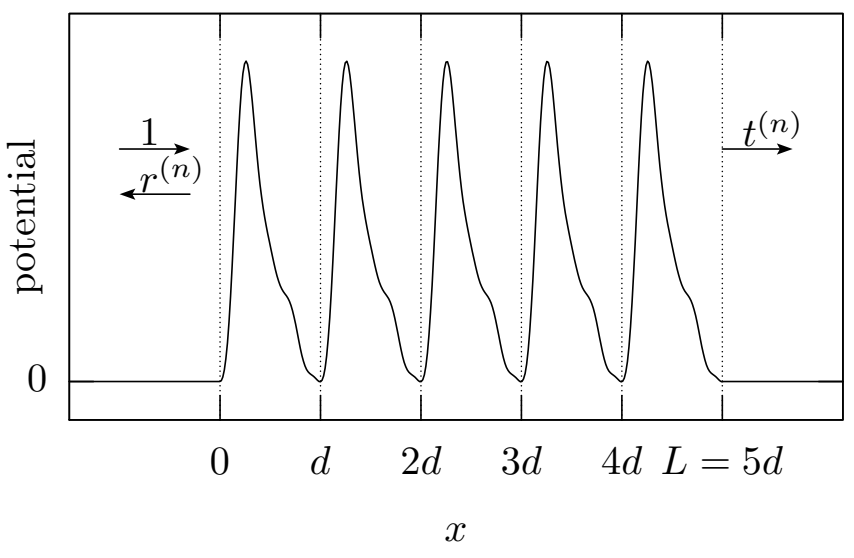

FIG. 1: Periodic potential (drawn for five periods) embedded between two infinite half-spaces. Arrows denote the wave function (plane waves).

Here $L$ is the length of the periodic system. Recent numerical calculations for finite periodic systems embedded in regions of constant potential showed that the equality in (10), does not hold in general27. At first glance it might seem paradox that the group velocity and the phase delay time, which are both based on a wave packet approach, lead to conflicting results.

The paper is organized as follows. In section $\llbracket$ we introduce the transfer matrix approach, which is used in III to calculate the resonant tunneling time and the corresponding velocity. In section IV Bloch's theorem is used together with the transfer matrix to decompose the wave function inside the finite periodic system (FPS) in two Bloch waves. It is shown that the velocity operator must have real expectation values at transmission resonances. Then the velocity expectation value is explicitly calculated. In section $\nabla$ we note that both velocity approaches lead to the same result and derive an upper bound for the velocity. The calculations show that Eq. (10) taken from Ref. 2 gives a wrong estimation when the unit cell transmission amplitude is small.

Further the special case when resonant tunneling velocity and group velocity are identical is discussed.

Our results are not restricted to a tight binding model, but are exact as long as only coherent transport is considered.

Finally, we apply the analytical results to a semiconductor superlattice and illustrate them with a compilation of graphs in section VI

\section{FINITE PERIODIC SYSTEMS}

Our one-dimensional model system consists of an $n$-fold periodic structure, extending from $x=0$ to $x=L=n d$ ( $d$ is the length of the unit cell), embedded between two semi-infinite half spaces with zero potential (Fig. 1).

Assuming a plane wave, $\exp (i k x)$, travelling from the 
left towards the finite periodic system (FPS), the wave function is given by

$$
\Psi(x)=\left\{\begin{array}{cc}
\exp (i k x)+r^{(n)} \exp (-i k x) & x \leq 0, \\
\Psi_{\text {FPS }}^{(n)}(x) & 0 \leq x \leq L=n d, \\
t^{(n)} \exp [i k(x-L)] & x \geq L,
\end{array}\right.
$$

where $k=\sqrt{2 m E} / \hbar$ is the electron wave vector in the semi-infinite half spaces, and $r^{(n)}$ and $t^{(n)}$ are the complex reflection and transmission coefficients of the $n$-fold periodic structure, respectively.

We start by briefly reviewing some important properties of one-dimensional finite periodic systems ${ }^{24.25}$. The wavefunctions at the left and right interface of a certain region, $\Psi_{L}$ and $\Psi_{R}$, respectively, are related by the transfer matrix $M$ through $^{28}\left(\begin{array}{c}A_{L}^{+} \\ A_{L}^{-}\end{array}\right)=M\left(\begin{array}{c}A_{R}^{+} \\ A_{R}^{-}\end{array}\right)$, where $\Psi_{L, R}=\left(\begin{array}{ll}1 & 1\end{array}\right)\left(\begin{array}{c}A_{L, R}^{+} \\ A_{L, R}^{-}\end{array}\right)=A_{L, R}^{+}+A_{L, R}^{-}$. Neglecting spin, the time reversal invariance and the conservation of the probability density current lead to the structure ${ }^{29}$ of the transfer matrix $M$

$$
M=\left(\begin{array}{cc}
a & b \\
b^{*} & a^{*}
\end{array}\right)
$$

where additionally $\operatorname{det} M=1$ holds $\left(x^{*}\right.$ denotes the complex conjugate of $x$ ). In terms of the transmission and reflection coefficients $t$ and $r$, the transfer matrix can be written $\mathrm{as}^{30}$

$$
M=\left(\begin{array}{cc}
1 / t & r^{*} / t^{*} \\
r / t & 1 / t^{*}
\end{array}\right)
$$

Since by construction the transfer matrix of a sequence of layers is the product of the transfer matrices of each layer, the transfer matrix of a potential consisting of $n$ periods is the $n$-th power of the transfer matrix of one period:

$$
M^{n}=\left(\begin{array}{cc}
a^{(n)} & b^{(n)} \\
b^{(n) *} & a^{(n) *}
\end{array}\right)
$$

For $n \geq 2, a^{(n)}$ and $b^{(n)}$ can be expanded to ${ }^{31}$

$$
\begin{aligned}
& a^{(n)}=a U_{n-1}(\operatorname{Re}\{a\})-U_{n-2}(\operatorname{Re}\{a\}), \\
& b^{(n)}=b U_{n-1}(\operatorname{Re}\{a\}),
\end{aligned}
$$

where $U_{n}(x)$ denote the Chebyshev Polynomials of the second kind. The transmission $T^{(n)}$ of any (field-free) $n$-fold periodic structure is given by $24,25,30,32,33$

$$
T^{(n)}=\left|a^{(n)}\right|^{-2}=\left[1+|b|^{2} U_{n-1}^{2}(\operatorname{Re}\{a\})\right]^{-1} .
$$

Resonances with $T^{(n)}=1$ occur if and only if $b^{(n)}=$ $b U_{n-1}(\operatorname{Re}\{a\})=0$. This leads to the condition of the transmission resonances 24.34

$$
T^{(n)}=1 \Leftarrow \operatorname{Re}\{a\}=\cos (j \pi / n), \quad j=1, \ldots, n-1 .
$$

The corresponding Bloch wave vectors $q_{j}^{\text {res }}$ are given by the condition $\operatorname{Re}\{a\}=\cos q d$ :

$$
q_{j}^{\text {res }}= \pm \frac{j \pi}{n d}, \quad j=1, \ldots, n-1 .
$$

Inserting Eq. (18) into (15) we get at resonance

$$
a^{(n)}\left(q_{j}^{\mathrm{res}}\right)=t^{(n)}\left(q_{j}^{\mathrm{res}}\right)=(-1)^{j} .
$$

\section{VELOCITY FROM TUNNELING TIME}

Recently the phase time for a system with $n$ periods, $\tau_{\text {phase }}^{(n)}$, has been calculated with the help of Eqs. (11), (13), and (15) 25.26.

$$
\begin{aligned}
& \tau_{\text {phase }}^{(n)}=\hbar T^{(n)}\left[\left(n-\frac{\operatorname{Re}\{a\}}{2} U_{2 n-1}(\operatorname{Re}\{a\})\right)\right. \\
& \left.\times \frac{\operatorname{Im}\{a\}}{1-\operatorname{Re}^{2}\{a\}} \frac{\partial \operatorname{Re}\{a\}}{\partial E}-\frac{1}{2} U_{2 n-1}(\operatorname{Re}\{a\}) \frac{\partial \operatorname{Im}\{a\}}{\partial E}\right]
\end{aligned}
$$

where $T^{(n)}$ is the transmission probability of the periodic structure given by Eq. (17). Here we are only interested in the phase time at resonance energies of the transmission, where the Bloch wave vector $q_{j}^{\text {res }}=j \pi / n d$ and $T^{(n)}=1$. For energies where $T^{(n)}=1$ the phase time is equal to the tunneling time as we discussed in the introduction and Eq. (21) reduces to the in-resonance phase time ${ }^{25}$ or resonant tunneling time:

$$
\tau_{\text {res }}^{(n)}=\hbar n \frac{\operatorname{Im}\{a\}}{1-\operatorname{Re}^{2}\{a\}} \frac{\partial \operatorname{Re}\{a\}}{\partial E} .
$$

Clearly, $\tau_{\text {res }}^{(n)}$ is proportional to the number of periods $n$. Using the natural definition

$$
v_{\text {res }}=L / \tau_{\text {res }}^{(n)},
$$

we get the following resonant tunneling velocity

$$
v_{\text {res }}=\hbar^{-1} d \frac{1-\operatorname{Re}^{2}\{a\}}{-\operatorname{Im}\{a\}}\left(-\frac{\partial \operatorname{Re}\{a\}}{\partial E}\right)^{-1},
$$

which does not depend on the number of periods $n$. For the sake of completeness we give the result in terms of the transmission amplitude $t=1 / a$ of the unit cell:

$$
v_{\text {res }}=\hbar^{-1} d \frac{|t|^{4}-\operatorname{Re}^{2}\{t\}}{\operatorname{Im}\{t\}}\left(-\frac{\partial \operatorname{Re}\{t\}}{\partial E}\right)^{-1} .
$$

Now it is interesting to compare this tunneling velocity $v_{\text {res }}$ to the group velocity $v_{g}$. From the dispersion relation $\operatorname{Re}\{a\}=\cos (q d)$ we obtain

$$
v_{g}=\left(\hbar \frac{\partial q}{\partial E}\right)^{-1}=\hbar^{-1} d \sqrt{1-\operatorname{Re}^{2}\{a\}}\left(-\frac{\partial \operatorname{Re}\{a\}}{\partial E}\right)^{-1} .
$$


Using Eq. (24), the resonant tunneling velocity and the group velocity are related by

$$
v_{\mathrm{res}}=\frac{\sqrt{1-\operatorname{Re}^{2}\{a\}}}{-\operatorname{Im}\{a\}} v_{g}=\frac{\sqrt{|t|^{4}-\operatorname{Re}^{2}\{t\}}}{\operatorname{Im}\{t\}} v_{g} .
$$

Note that the equations for the resonant tunneling time and the resonant velocity are only meaningful if they are evaluated at energies where the FPS has a transmission probability of unity.

Nevertheless, by increasing the number of periods towards infinity the discrete curves tend to continuous ones, just like the dispersion relation does.

\section{EXPECTATION VALUE OF VELOCITY OPERATOR}

In the following we calculate the expectation value of the velocity operator applied to the exact wave function inside the FPS.

\section{A. Decomposition of $\Psi$ into two Bloch waves}

In 1929 Bloch calculated the eigenfunctions of the Schrodinger equation for crystal lattices 35 . He modelled the lattice by a periodic potential which spans the whole space. Using group theory together with periodic (Born von Kármán) boundary conditions he proved that the base solutions of the Schrödinger equation for a lattice potential, i.e. $V(\mathbf{x})=V\left(\mathbf{x}+\mathbf{R}_{i}\right)$, where $\mathbf{R}_{i}$ is any lattice vector, are of the form:

$$
\Psi_{\mathbf{q}}^{B}(\mathbf{x})=u_{\mathbf{q}}(\mathbf{x}) \exp (i \mathbf{q} \cdot \mathbf{x})
$$

Here $\mathbf{q}$ is in modern terms a reciprocal lattice vector and

$$
u_{\mathbf{q}}(\mathbf{x})=u_{\mathbf{q}}\left(\mathbf{x}+\mathbf{R}_{i}\right)
$$

is a lattice periodic function. This fact is well known as Bloch's theorem. Since Bloch's theorem was originally derived for an infinite domain, this infinite domain is sometimes considered as necessary.

Therefore, we will start by briefly showing that Bloch's theorem gives also the exact wave functions in finite systems.

Mathematically spoken, for the case of the infinite periodic potential $V: \mathbb{R}^{3} \rightarrow \mathbb{R}$ considered by Bloch, the domain of the Schrödinger differential equation and its solutions $\Psi_{\mathbf{q}}^{B}$ is $D=\mathbb{R}^{3}$. If we reduce the domain of the periodic potential (and thus of the Schrödinger equation) to any finite subdomain $\tilde{D} \subset \mathbb{R}^{3}$ and choose an arbitrary potential in the domain $\mathbb{R}^{3} \backslash \tilde{D}$ the base solutions of the differential equation inside $\tilde{D}$ are not changed. The physical consequence is that despite the loss of the global translation invariance of the Hamiltonian in a finite system, the wave functions are still exactly given by superpositions of Bloch waves, Eq. (28).

If we neglect spin the time inversion symmetry of the Hamiltonian leads to Kramers degeneracy, i.e. $E(\mathbf{q})=$ $E(-\mathbf{q})$

Therefore, the two linear independent solutions of Schrödinger's equation for the one-dimensional periodic potential in a finite domain are given by $\Psi_{q}^{B}$ and $\left(\Psi_{q}^{B}\right)^{*}(x)=\Psi_{-q}^{B}(x)$. Having clarified this we use the following ansatz for the wave function in any onedimensional $n$-fold periodic system inside an allowed band:

$$
\begin{aligned}
\Psi_{\mathrm{FPS}}^{(n)}(x)= & \alpha_{q}^{(n)} u_{q}(x) \exp (i q x) \\
& +\alpha_{-q}^{(n)} u_{q}^{*}(x) \exp (-i q x), \quad 0 \leq x \leq n d \\
u_{q}(x+d) & =u_{q}(x), \quad u_{q}^{*}(x)=u_{-q}(x) .
\end{aligned}
$$

The dimensionless coefficients $\alpha_{q}^{(n)}$ and $\alpha_{-q}^{(n)}$ are determined by inital value conditions, i.e. by $\Psi(0)$ and $\Psi^{\prime}(0)$. In the following we choose the $u_{q}(x)$ to be normalized

$$
\int_{0}^{d} d x u_{q}^{*}(x) u_{q}(x)=d /(2 \pi)
$$

\section{B. The velocity operator has real eigenvalues at transmission resonances}

Any physical state is represented by a wave function which is an element of the Hilbert space of the square integrable functions $\mathcal{H}=L^{2}\left(\mathbb{R}^{3}\right)$. The Hermiticity of the velocity (and the momentum) operator comes from the fact that the normalization $\langle\Psi \mid \Psi\rangle=1$ leads to $\Psi(\mathbf{x})=0$ as $|\mathbf{x}| \rightarrow \infty$.

The states we consider, Eq. (11), are scattering states which do not belong to $L^{2}\left(\mathbb{R}^{3}\right)$. Therefore, we can not expect that the velocity operator applied to any finite subdomain is Hermitian. Nevertheless, we show that the expectation values of the velocity operator inside the periodic structure are real for resonant tunneling states.

The calculation is restricted to the one-dimensional region between $x=0$ and $x=L$ inside the FPS. The expectation value of the velocity of any state $|\Psi\rangle$ is given by $\langle\hat{v}\rangle=\langle\Psi|\hat{v}| \Psi\rangle /\langle\Psi \mid \Psi\rangle$. In our case the numerator can be denoted as $\left\langle\Psi_{\text {FPS }}^{(n)}\left|\hat{v} \hat{P}_{\text {FPS }}\right| \Psi_{\text {FPS }}^{(n)}\right\rangle$, where $\hat{P}_{\text {FPS }}$ is the projection operator onto the space region of the FPS,

$$
\hat{P}_{\mathrm{FPS}}=\int_{0}^{L} d x|x\rangle\langle x| .
$$

Partial integration yields:

$$
\begin{aligned}
\left\langle\Psi_{\mathrm{FPS}}^{(n)}\left|\hat{v} \hat{P}_{\mathrm{FPS}}\right| \Psi_{\mathrm{FPS}}^{(n)}\right\rangle & =\left.\left(\frac{-i h}{m}\right)\left|\Psi_{\mathrm{FPS}}^{(n)}\right|^{2}\right|_{0} ^{L}+ \\
& \left\langle\Psi_{\mathrm{FPS}}^{(n)}\left|\hat{v} \hat{P}_{\mathrm{FPS}}\right| \Psi_{\mathrm{FPS}}^{(n)}\right\rangle^{*} .
\end{aligned}
$$


At resonances with $\left|t^{(n)}\right|=1$ the term $\left.\left|\Psi_{\text {FPS }}^{(n)}\right|^{2}\right|_{0} ^{L}$ vanishes and we obtain:

$$
\left\langle\Psi_{\mathrm{FPS}}^{(n)}\left|\hat{v} \hat{P}_{\mathrm{FPS}}\right| \Psi_{\mathrm{FPS}}^{(n)}\right\rangle=\left\langle\Psi_{\mathrm{FPS}}^{(n)}\left|\hat{v} \hat{P}_{\mathrm{FPS}}\right| \Psi_{\mathrm{FPS}}^{(n)}\right\rangle^{*}
$$

i.e., the velocity has a real value.

\section{Expectation value of the velocity at resonance}

The expectation value of the velocity in the $n$-periodic system at resonance is given by

$$
\left\langle\hat{v}_{\mathrm{FPS}}^{(n)}\right\rangle=\frac{\left\langle\Psi_{\mathrm{FPS}}^{(n)}\left|\hat{v} \hat{P}_{\mathrm{FPS}}\right| \Psi_{\mathrm{FPS}}^{(n)}\right\rangle}{\left\langle\Psi_{\mathrm{FPS}}^{(n)}\left|\hat{P}_{\mathrm{FPS}}\right| \Psi_{\mathrm{FPS}}^{(n)}\right\rangle} .
$$

With the help of Eqs. (30) and (31) the numerator is given by

$$
\left\langle\Psi_{\mathrm{FPS}}^{(n)}\left|\hat{v} \hat{P}_{\mathrm{FPS}}\right| \Psi_{\mathrm{FPS}}^{(n)}\right\rangle=\frac{L}{2 \pi}\left(\left|\alpha_{q}^{(n)}\right|^{2}-\left|\alpha_{-q}^{(n)}\right|^{2}\right) v_{g}(q) .
$$

The denominator of (35) is given by

$$
\left\langle\Psi_{\mathrm{FPS}}^{(n)}\left|\hat{P}_{\mathrm{FPS}}\right| \Psi_{\mathrm{FPS}}^{(n)}\right\rangle=\frac{L}{2 \pi}\left(\left|\alpha_{q}^{(n)}\right|^{2}+\left|\alpha_{-q}^{(n)}\right|^{2}\right) .
$$

A detailed derivation of both equations is given in appendix $\mathrm{A}$ and $\mathrm{B}$ respectively. We stress that these simple expressions hold only in the resonant case. From Eqs. (35) to (37) we obtain the velocity expectation value at resonance:

$$
\left\langle\hat{v}_{\mathrm{FPS}}^{(n)}\right\rangle=\frac{\left|\alpha_{q}^{(n)}\right|^{2}-\left|\alpha_{-q}^{(n)}\right|^{2}}{\left|\alpha_{q}^{(n)}\right|^{2}+\left|\alpha_{-q}^{(n)}\right|^{2}} v_{g}=\frac{1-\left|\alpha_{-q}^{(n)} / \alpha_{q}^{(n)}\right|^{2}}{1+\left|\alpha_{-q}^{(n)} / \alpha_{q}^{(n)}\right|^{2}} v_{g} .
$$

An interpretation of this formula will be given in the next section.

\section{Determination of Bloch coefficients}

Next we have to determine the ratio $\left|\alpha_{-q}^{(n)} / \alpha_{q}^{(n)}\right|$. For the calculation of the expectation value it would be sufficient to consider only the wave function at resonance, nevertheless we will derive a more general result which is valid for any $q$ value.

We rewrite the ansatz for the wave function (30) in a reduced form

$$
\begin{aligned}
\Psi_{\mathrm{FPS}}^{(n)}(x) & =\tilde{u}_{q}(x) \exp (i q x)+\tilde{\alpha}_{-q}^{(n)} \tilde{u}_{q}^{*}(x) \exp (-i q x) \\
\tilde{u}_{q}(x) & =\alpha_{q}^{(n)} u_{q}(x) \\
\tilde{\alpha}_{-q}^{(n)} & =\alpha_{-q}^{(n)} /\left(\alpha_{q}^{(n)}\right)^{*}
\end{aligned}
$$

By formally replacing $q$ with $-q$ and taking the complex conjugate in Eq. (39c)

$$
\tilde{\alpha}_{-q}^{(n)}=1 /\left(\tilde{\alpha}_{q}^{(n)}\right)^{*}
$$

is obtained.

As stated above, the remaining coefficient $\tilde{\alpha}_{-q}^{(n)}$ is determined by the initial values $\Psi(0)$ and $\Psi^{\prime}(0)$, obtained from the continuity of the wave function and the probability current density across the boundary at $x=0$. To simplify the algebra, we make use of the fact that these continuity conditions are inherently incorporated in the transfer matrix approach. Therefore, to determine $\tilde{\alpha}_{-q}^{(n)}$ we use the continuity of the wavefunction at $x=0$ and two different representations of $\Psi_{\text {FPS }}^{(n)}$ at $x=d$. This way the derivative of the wave function is not needed in an explicit form. ${ }^{36}$ The continuity of the wavefunction (11) at $x=0$ leads to

$$
1+r^{(n)}=\tilde{u}_{q}(0)+\tilde{\alpha}_{-q}^{(n)} \tilde{u}_{q}^{*}(0) .
$$

With Eq. 39a the periodicity of the $\tilde{u}_{q}(x)$ gives the wavefunction at $x=d$ :

$$
\Psi_{\mathrm{FPS}}^{(n)}(d)=\tilde{u}_{q}(0) \exp (i q d)+\tilde{\alpha}_{q}^{(n)} \tilde{u}_{-q}^{*}(0) \exp (-i q d)
$$

On the other hand $\Psi_{\text {FPS }}^{(n)}(d)$ can be obtained through the transfer matrix (12) of the unit cell:

$$
\begin{aligned}
\Psi_{\mathrm{FPS}}^{(n)}(d) & =\left(\begin{array}{ll}
1 & 1
\end{array}\right) M^{-1}\left(\begin{array}{c}
1 \\
r^{(n)}
\end{array}\right)= \\
& =a^{*}-b^{*}+r^{(n)}(a-b) .
\end{aligned}
$$

Again, we note that the continuity of the probability current density is fully incorporated in this treatment in the reflection coefficient $r^{(n)}$. Equations (40), and (41) together with (42) form two non-linear equations in $\tilde{u}_{q}(0)$ and $\tilde{\alpha}_{-q}^{(n)}$. The solution for $\tilde{\alpha}_{-q}^{(n)}$ is given by

$$
\tilde{\alpha}_{-q}^{(n)}=\frac{a^{*}-b^{*}-\xi+r^{(n)}(a-b-\xi)}{a-b-\xi+r^{(n) *}\left(a^{*}-b^{*}-\xi\right)},
$$

where $\xi=\exp (i q d)$ is introduced. From Eqs. (13) to (16) we obtain

$$
r^{(n)}=\frac{b^{(n) *}}{a^{(n)}}=\frac{b^{*} U_{n-1}(\operatorname{Re}\{a\})}{a U_{n-1}(\operatorname{Re}\{a\})-U_{n-2}(\operatorname{Re}\{a\})} .
$$

Inserting into (43) we get after some algebra

$$
\tilde{\alpha}_{-q}^{(n)}=\frac{a^{*}-b^{*}-\xi}{a-b-\xi} \frac{a^{(n) *}}{a^{(n)}}=\frac{a^{*}-b^{*}-\xi}{a-b-\xi} \frac{t^{(n)}}{t^{(n) *}} .
$$

Consistent with (39d), $\tilde{\alpha}_{-q}^{(n)}=1 /\left(\tilde{\alpha}_{q}^{(n)}\right)^{*}$ is fulfilled. In appendix C] Eq. (45) is used to calculate $\tilde{u}_{q}(x)$ and 
$\Psi_{\text {FPS }}^{(n)}(x)$. Further we prove in appendix D1 the interesting identity

$$
\left\langle\Psi_{\mathrm{FPS}}^{(n)}\left|\hat{v} \hat{P}_{\mathrm{FPS}}\right| \Psi_{\mathrm{FPS}}^{(n)}\right\rangle=j_{i n} L
$$

where $j_{\text {in }}$ is the incident probability current. Eq. (46) is used for the calculation of $\alpha_{-q}^{(n)}$ and $\alpha_{q}^{(n)}$ in appendix D3

As a consequence of (45) the absolute value of $\tilde{\alpha}_{-q}^{(n)}$ does not depend on $n$ :

$$
\left|\tilde{\alpha}_{-q}^{(n)}\right|=\left|\frac{a^{*}-b^{*}-\xi}{a-b-\xi}\right| .
$$

This shows that the ratio of the amplitude of the left and right going Bloch wave does not depend on the number of periods and is a property of the unit cell only.

Using $\operatorname{Re}\{a\}=\cos q d,|a|^{2}-|b|^{2}=1$, and $\xi=\exp (i q d)$, the absolute value squared of $\tilde{\alpha}_{-q}^{(n)}$ can be simplified to:

$$
\left|\tilde{\alpha}_{-q}^{(n)}\right|^{2}=\frac{\operatorname{Im}\{a\}+\sqrt{1-\operatorname{Re}^{2}\{a\}}}{\operatorname{Im}\{a\}-\sqrt{1-\operatorname{Re}^{2}\{a\}}} .
$$

This implies that at the edges of the allowed band $\left|\tilde{\alpha}_{-q}^{(n)}\right|$ reaches unity:

$$
q=(0, \pi / d)+2 p \pi / d \Leftrightarrow|\operatorname{Re}\{a\}|=1 \Leftrightarrow\left|\tilde{\alpha}_{-q}^{(n)}\right|=1 .
$$

Taking $\left|\tilde{\alpha}_{-q}^{(n)}\right|=\left|\alpha_{-q}^{(n)} / \alpha_{q}^{(n)}\right|$ into consideration, inserting (48) into (38) gives finally

$$
\left\langle\hat{v}_{\mathrm{FPS}}^{(n)}\right\rangle=\frac{1-\left|\tilde{\alpha}_{-q}^{(n)}\right|^{2}}{1+\left|\tilde{\alpha}_{-q}^{(n)}\right|^{2}} v_{g}=\frac{\sqrt{1-\operatorname{Re}^{2}\{a\}}}{-\operatorname{Im}\{a\}} v_{g} .
$$

However, this result is not true for the off-resonant case when the first equality does not hold. Nevertheless, the behaviour of $\left\langle\hat{v}_{\mathrm{FPS}}^{(n)}\right\rangle$ as a continuous function of $E$ or $q$ is of interest, since any rational multiple of $\pi / d$ can be obtained as $q_{\text {res }}$, by choosing proper values for $j$ and $n$, see Eq. (19).

For the sake of completeness, in the resonant case, where $q_{\text {res }}$ is given by Eq. (19), and $t^{(n)}= \pm 1$, Eq. (45) can be simplified to

$$
\tilde{\alpha}_{-q_{\mathrm{res}}}^{(n)}=\frac{a^{*}-b^{*}-\xi}{a-b-\xi} .
$$

\section{IDENTITY OF TUNNELING TIME APPROACH AND VELOCITY EXPECTATION VALUE APPROACH}

Comparing Eqs. (27) and (50), we get the important identity

$$
\left\langle\hat{v}_{\mathrm{FPS}}^{(n)}\right\rangle=v_{\mathrm{res}} .
$$

Consequently, the tunneling time approach and the velocity expectation value approach are physically equivalent at resonance. We use the term $v_{\text {res }}$ as the electron velocity in resonant tunneling through a FPS in the following. From the first identity in Eq. (50) follows, that the electron velocity is bounded above by the group velocity $v_{g}$ :

$$
v_{\text {res }} \leq\left|v_{g}\right| .
$$

Here the absolute value occurs, since the group velocity can also take negative values for positive $q$, e.g. for bands where the energy maximum is at $q=0$, while the resonant velocity is always positive.

\section{A. Upper bound for the resonant tunneling velocity}

In fact, by squaring the outer equality in (150), an improved inequality compared to (53) can be derived. We use that $|a|^{2}=|t|^{-2} \geq 1$. Additionally, inside an allowed band $\operatorname{Re}^{2}\{a\} \leq 1$ holds. Therefore, $0 \leq$ $1-\operatorname{Re}^{2}\{a\} \leq \operatorname{Im}^{2}\{a\}$. Now making use of the simple inequality $\frac{x}{y} \leq \frac{x+z}{y+z}$ that holds for $0 \leq x \leq y$ and $0 \leq z$ we obtain

$$
\frac{1-\operatorname{Re}^{2}\{a\}}{\operatorname{Im}^{2}\{a\}} \leq \frac{1}{\operatorname{Im}^{2}\{a\}+\operatorname{Re}^{2}\{a\}}=|t|^{2} .
$$

Finally, this proofs

$$
v_{\text {res }} \leq|t|\left|v_{g}\right| .
$$

In both formulas the equality holds for $\operatorname{Re}\{a\}=0$, i.e. for $q=\pi / 2 d$, which is in the middle of the band in $q$-space. This proves that the resonant tunneling velocity can be much smaller than the group velocity, given a sufficient small transmission amplitude of the unit cell. Furthermore, towards the band edges where $\operatorname{Re}\{a\}$ approaches \pm 1 , the ratio $v_{\text {res }} / v_{g}$ vanishes, see Eq. (50). Comparing with Eq. (10), taken from Ref. 2, we conclude that the matching of the wave functions at the ends of the system can reduce the velocity considerably.

\section{B. Special case: Identity between $v_{\text {res }}$ and $\left|v_{g}\right|$}

From the outer equality in Eq. (50) we can conclude that the equality between $v_{\text {res }}$ and $\left|v_{g}\right|$ holds if and only if $|a|=|t|^{-1}=1$, i.e. at energies $E^{\prime}$ with $\left|t\left(E^{\prime}\right)\right|=1$ of the unit cell of the periodic system:

$$
v_{\text {res }}\left(E^{\prime}\right)=\left|v_{g}\left(E^{\prime}\right)\right| \Leftrightarrow\left|t\left(E^{\prime}\right)\right|=1 .
$$

A unit cell which is formed by a symmetric double barrier resonant tunneling structure ${ }^{37.38}$ possesses the property $\left|t\left(E^{\prime}\right)\right|=1$ at each transmission resonance energy $E^{\prime}=$ $E_{n}$. For this unit cell type the resonant tunneling velocity 
$v_{\text {res }}$ equals the magnitude of the group velocity $v_{g}$ at all energies $E_{n}$. For energies different from the $E_{n}, v_{\text {res }}$ is smaller than $\left|v_{g}\right|$. The only unit cell where $v_{\text {res }}$ equals $v_{g}$ for all energies is the trivial unit cell with $V(x)=0$, for $0 \leq x \leq d$, since it has $|t(E)|=1$ for all energies.

Further we can derive conditions from Eq. (50) for the identity between $v_{\text {res }}$ and $\left|v_{g}\right|$, in terms of the coefficients $\alpha_{q}^{(n)}, \alpha_{-q}^{(n)}$ :

$$
\begin{aligned}
& v_{\text {res }}=v_{g} \Leftrightarrow \alpha_{-q}^{(n)}=0, \\
& v_{\text {res }}=-v_{g} \Leftrightarrow \alpha_{q}^{(n)}=0 .
\end{aligned}
$$

The resonant tunneling velocity equals the magnitude of the group velocity if and only if the wave function has only one Bloch component.

Considering Eq. (56) this can also be written as

$$
\alpha_{q}^{(n)}=0 \vee \alpha_{-q}^{(n)}=0 \Leftrightarrow|t|=1,
$$

yielding that the wave function inside a finite periodic potential consists of only one Bloch wave if and only if the transmission of the unit cell reaches unity. Therefore, we obtain the picture, that the Bloch wave moving into the left direction is built up by a coherent superposition of the reflected parts of waves moving into the right direction.

This solves also the puzzle we mentioned in the introduction, namely, that the phase delay time and the group velocity both originate from a wave packet analysis, but lead to different results. Often the group velocity is used when in good approximation no reflection occurs inside the medium. In contrast, here, in the case that $|t| \neq 1$, there is reflection in each period. These reflections are the origin of the reduced velocity, compared to the group velocity.

\section{GAAS/ALGAAS SUPERLATTICE}

All presented results are valid for arbitrary unit cells unless otherwise noted. In this section the results are applied to a periodic semiconductor heterostructure. Due to its widespread use we choose a GaAs/AlGaAs superlattice (SL). Denoting the barriers (AlGaAs) with B and the well regions (GaAs) with $\mathrm{A}$, we can give a short notation for the potential with $n$ periods: $(\mathrm{BA})^{n}$. Assuming a stepwise constant potential function in each layer the transfer matrix elements can be calculated analytically. To keep the focus on the main topic, we will use the following simplifications: (i) the effective mass $m^{*}$ is approximated to be the same in GaAs and in AlGaAs, and (ii) the effective mass does not depend on the energy. This simplifications can be avoided but this is left for future work. We present results for the lowest miniband. The analogous calculations for higher minibands will be discussed elsewhere.

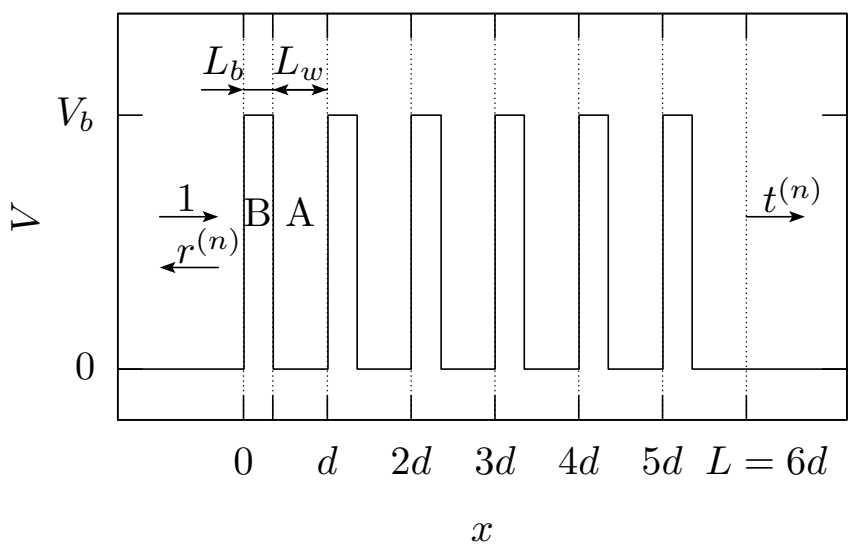

FIG. 2: Schematic potential of a superlattice of the form $(\mathrm{BA})^{n}$. The number of periods $n$ is chosen to be 6 .

For a SL we get the following expressions for the matrix elements 25

$$
\begin{aligned}
\operatorname{Re}\left\{a_{S L}\right\} & =\cosh \kappa L_{b} \cos k L_{w}-c_{2} \sinh \kappa L_{b} \sin k L_{w} \\
\operatorname{Im}\left\{a_{S L}\right\} & =-\cosh \kappa L_{b} \sin k L_{w}-c_{2} \sinh \kappa L_{b} \cos k L_{w}, \\
b_{S L} & =i c_{1} \sinh \kappa L_{b} \exp k L_{w} .
\end{aligned}
$$

Here $L_{b}$ and $L_{w}$ are the thicknesses of the barrier and the well layers,

$$
\kappa=\hbar^{-1}\left[2 m^{*}\left(V_{b}-E\right)\right]^{1 / 2}, k=\hbar^{-1}\left(2 m^{*} E\right)^{1 / 2},
$$

are the decaying electron wave vector in the barrier layer and the electron wave vector in the well layer, respectively and

$$
c_{1,2}=\frac{1}{2}\left(k \kappa^{-1} \pm \kappa k^{-1}\right) .
$$

The energy and the conduction band offset between GaAs and AlGaAs are denoted $E$ and $V_{b}$, respectively.

The following SL parameters are choosen to be identical or nearly identical to systems which were studied experimentally and theoretically in the past ${ }^{25.27 .34 .39}$.

For the barrier and the well width we choose $L_{b}=$ $2.5 \mathrm{~nm}$ and $L_{w}=6.5 \mathrm{~nm}$, resp. The barriers consist of $\mathrm{Al}_{.3} \mathrm{Ga} .7 \mathrm{As}$, the wells of GaAs. The number of periods is $n=6$. A schematic of the resulting conduction band is drawn in Fig. 2 The calculations are performed for the $\Gamma$-valley of the conduction band for a temperature of $4 \mathrm{~K}$. As the effective electron masses in both, GaAs and $\mathrm{Al}_{.3} \mathrm{Ga}_{.7} \mathrm{As}$, we choose $m^{*}=0.072 m_{0}$, where $m_{0}$ is the free electron mass. The conduction band offset is $V_{b}=288 \mathrm{meV}$.

Because of the strong interdependencies between the dispersion relation, transmission probability, group and resonant tunneling velocity, and the ratio of the amplitudes of the $+q$ and $-q$ Bloch waves, all are shown together in Fig. 3] All variables are plotted on the left as a 


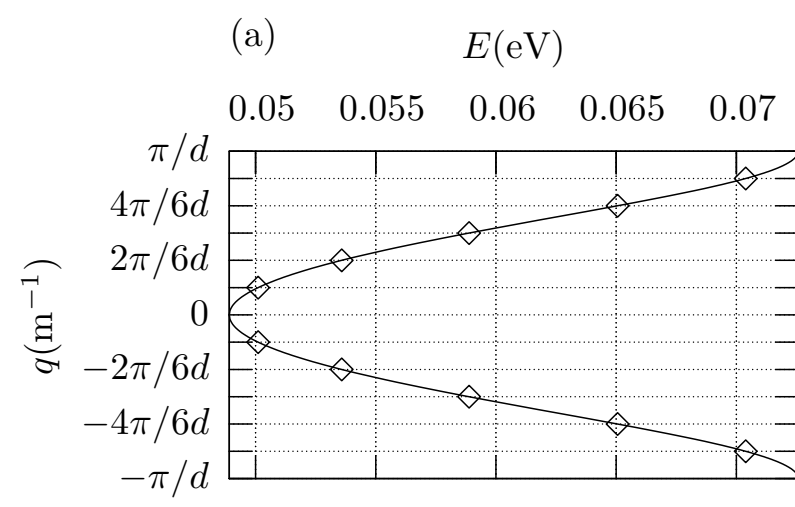

(b)

$\quad q\left(\mathrm{~m}^{-1}\right)$
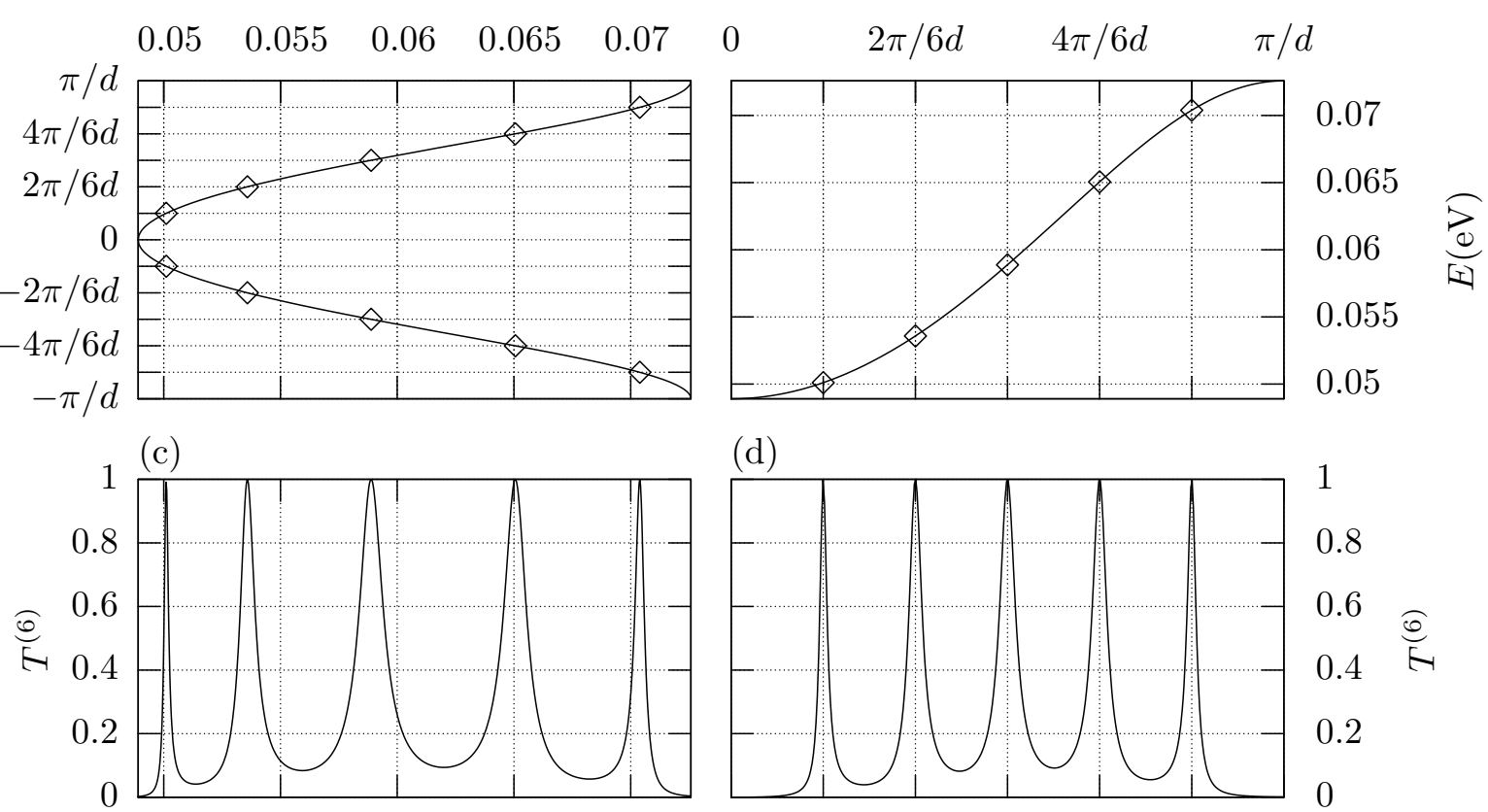

(d)
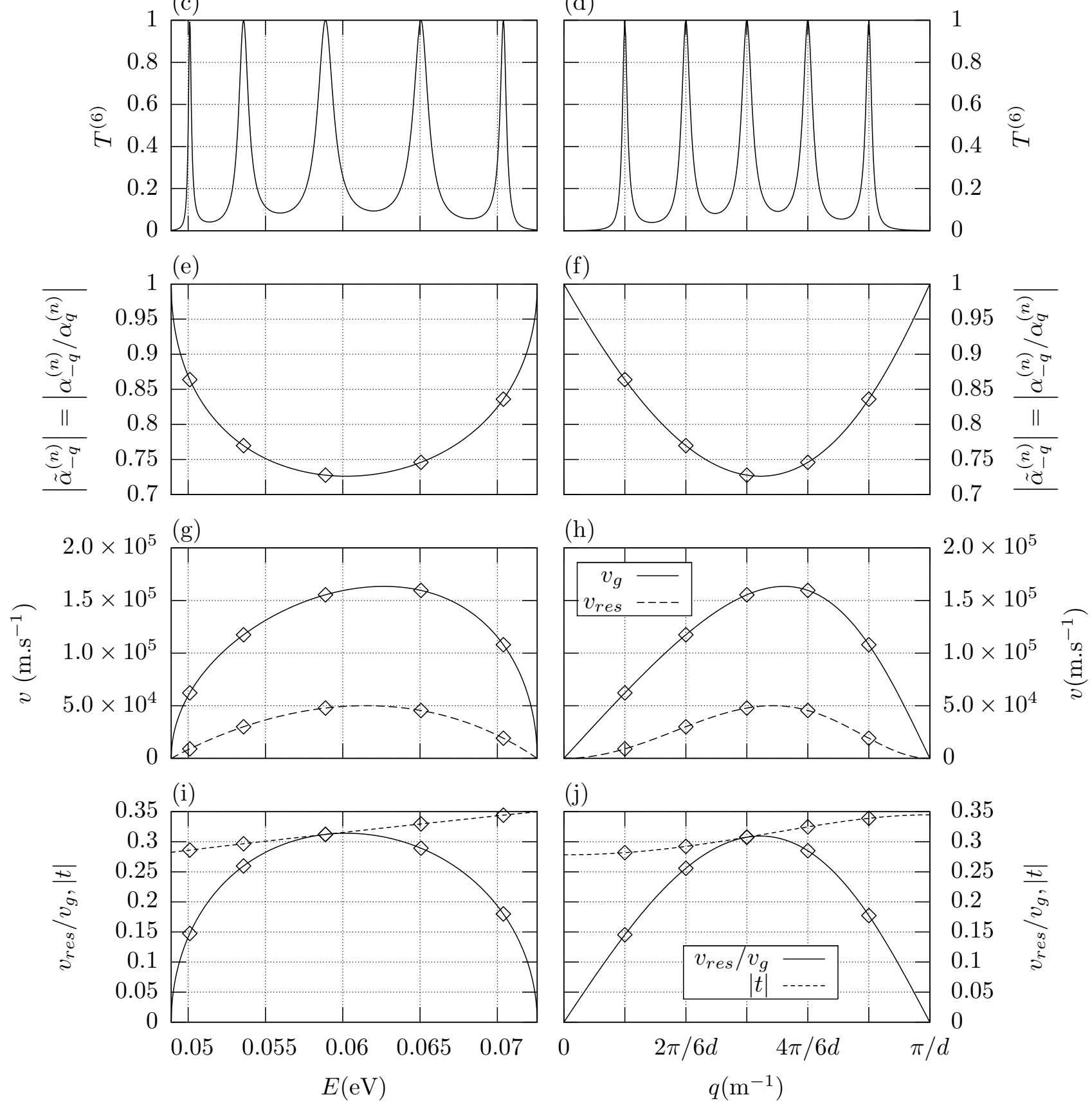

FIG. 3: Shown are relevant physical properties of superlattices. In the left column all functions are plotted vs. energy $E$, in the right column vs. Bloch wave vector $q$. Diamonds mark the positions of the resonant levels for $n=6$ periods (band structure given in Fig. 2). By changing the number of periods $n$, the lines connecting the diamonds are formed. The dispersion relation, $q(E)$ (and $E(q)$ ), the transmission probability, $T^{(6)}$, the normalized coefficient of the backwards propagating Bloch wave, $\left|\tilde{\alpha}_{-q}^{(n)}\right|$, the group velocity, $v_{g}$, and the resonant tunneling velocity $v_{\text {res }}$, and the ratio of resonant tunneling and group velocity of the periodic system together with the magnitude of the unit cell transmission amplitude, $|t|$, are shown. Details are given in the text. 
function of the energy $E$ and on the right as a function of the Bloch wave vector $q$.

For the first miniband the resonance energies are given by the smallest solutions $E_{j}$ of Eqs. (18) and (60a), i.e., by solving the transcendental equation

$$
\cos (j \pi / n)=\cosh \kappa L_{b} \cos k L_{w}-c_{2} \sinh \kappa L_{b} \sin k L_{w} .
$$

The diamonds mark the values at the resonance energies for the system with $n=6$ periods. Changing the number of periods $n$ results in values that lie on the lines which connect the diamonds. In the limit $n \rightarrow \infty$ one would also obtain the continuous lines.

Figure 3 (a) shows $q(E)$, and Fig. 3 (b) shows its inverse, the dispersion relation $E(q)$. The dispersion relation is obtained from $\operatorname{Re}\left\{a_{S L}\right\}=\cos q d$, where $\operatorname{Re}\left\{a_{S L}\right\}$ is given by Eq. 60a .

In Fig. [3 (c) and (d), the transmission probability, $T^{(6)}$, Eq. (17), is plotted.

From the plots of $E(q)$ and $T^{(6)}(q)$ the equidistant behaviour of the resonant levels, Eq. (19), in the $q$-space can be nicely seen. Of course, due to the nonlinear dispersion $E(q)$, this behaviour is lost in energy-space.

Next, in Fig. B(e) and (f), the magnitude of the amplitude ratio of the $-q$ and $+q$ Bloch waves, $\left|\tilde{\alpha}_{-q}^{(n)}\right|$, Eq. (47), is shown. At the miniband edges this ratio reaches exactly unity, and it drops to a minimum around the middle of the miniband.

Figure 3 (g) and (h) show the group velocity, $v_{g}$, Eq. (26) (solid line), and the resonant tunneling velocity, $v_{\text {res }}$, Eq. (24) (dashed line). For the $n=6$ case, the group velocity reaches its maximum in the fourth resonance, while the resonant tunneling velocity reaches its maximum in the third resonance. This shows again the fundamental difference between both velocities. It is interesting to note that the slope of $v_{\text {res }}(q)$ is zero at $q=0, \pi / d$, while the slope of $v_{g}$ has its maximum value there.

In Fig. 3 (i) and (j), the ratio $v_{\text {res }} / v_{g}$ and the magnitude of the unit cell transmission amplitude, $|t|$, are plotted. The velocity ratio is always smaller than the single cell transmission, except for $q=\pi / 2 d$ when both are equal, see Eq. (55). For the given parameters the velocity ratio is below one third.

\section{CONCLUSIONS}

We calculated the resonant tunneling electron velocity in finite periodic structures embedded in regions of constant potential in two different ways and proved their identity.

The first method was based on the fact that $\left|t^{(n)}\right|=1$ leads to a coincidence of all tunneling time definitions, turning up in the literature. We used the phase time $\tau_{\text {phase }}=\hbar \partial \arg t(E) / \partial E$ and the natural definition $v=$
$L / \tau$ to calculate the velocity in terms of the unit cell transfer matrix elements and the unit cell transmission amplitude, respectively, yielding Eqs. (24) and (25).

Bloch's theorem was combined with the transfer matrix approach to separate the wave function into two Bloch waves, which propagate in opposite directions, Eq. (39a) together with either Eq. (47) or Eq. (48). After proving that the velocity operator is real at resonance, we calculated its expectation value, Eq. (50), as the second method.

Both results are completely identical, showing the physical equivalence of the two approaches. The resonant tunneling velocity is in any case smaller or equal to the group velocity. In addition, the resonant tunneling velocity is smaller than or equal to the product of the group velocity and the magnitude of the transmission amplitude of the unit cell. Thus for unit cells with a small transmission amplitude both velocities can differ considerably. We discussed that the Bloch wave moving in the opposite direction of the incident electrons is due to reflections inside all unit cells. These reflections are the reason for the reduced velocity compared to the group velocity. At energies, where the unit cell has a transmission of unity, only one Bloch wave remains. Consequently the resonant tunneling velocity equals the group velocity.

The group velocity is often used as the speed of the electrons inside a superlattice. Our result points out that taking into account the boundary conditions reduces the actual velocity considerably.

In this context the often used relation "mean free path equals group velocity times scattering time" seems questionable. We suggest that in mesoscopic structures, where the mean free path exceeds the length of the periodic structure it should be replaced by "mean free path equals resonant tunneling velocity multiplied by scattering time". The presented results support the numerical findings of Ref. 27.

We used electron waves throughout this paper. In analogy the main results hold for the propagation of light waves, of phonons waves and so on in periodic structures.

\section{Acknowledgments}

Finally it is a pleasure to thank A. Wacker, J. Burgdörfer and R. Dirl for interesting discussions and valuable comments and to acknowledge financial support by the Austrian Science Fonds (FWF) Grant No. Z24.

\section{APPENDIX A: EVALUATION OF $\left\langle\Psi_{\text {FPS }}^{(n)}\left|\hat{v} \hat{P}_{\text {FPS }}\right| \Psi_{\text {FPS }}^{(n)}\right\rangle$}

Expanding the integral with the help of Eqs. (30) and (31) first leads to 


$$
\begin{aligned}
\left\langle\Psi_{\mathrm{FPS}}^{(n)}\left|\hat{v} \hat{P}_{\mathrm{FPS}}\right| \Psi_{\mathrm{FPS}}^{(n)}\right\rangle= & \left|\alpha_{q}^{(n)}\right|^{2}\left\langle\Psi_{q}^{B}\left|\hat{v} \hat{P}_{\mathrm{FPS}}\right| \Psi_{q}^{B}\right\rangle+\left|\alpha_{-q}^{(n)}\right|^{2}\left\langle\Psi_{-q}^{B}\left|\hat{v} \hat{P}_{\mathrm{FPS}}\right| \Psi_{-q}^{B}\right\rangle \\
& -\frac{i \hbar}{m}\left(\alpha_{q}^{(n)} \alpha_{-q}^{(n) *} \int_{0}^{L} d x u_{q}(x) e^{i q x} \frac{\partial}{\partial x}\left(u_{q}(x) e^{i q x}\right)+\text { c.c. }\right) .
\end{aligned}
$$

In resonance the Bloch wave vector $q$ is given by (19), i.e., $q_{j}^{\text {res }}=j \pi / L$. For these $q_{j}^{\text {res }}$ the last integral vanishes:

$$
\int_{0}^{L} d x u_{q}(x) e^{i q x} \frac{\partial}{\partial x}\left(u_{q}(x) e^{i q x}\right)=\left.\frac{1}{2} u_{q}^{2}(x) e^{i 2 q x}\right|_{0} ^{L}=0,
$$

due to the periodicity of both, the $u_{q}(x)$ and the complex exponential function. Using the fact $\frac{40}{}$ that

$$
\left\langle\Psi_{ \pm q}^{B}|\hat{v}| \Psi_{ \pm q}^{B}\right\rangle /\left\langle\Psi_{ \pm q}^{B} \mid \Psi_{ \pm q}^{B}\right\rangle=v_{g}( \pm q)= \pm v_{g}(q),
$$

the normalization (31) leads finally to Eq. (36).

\section{APPENDIX B: EVALUATION OF $\left\langle\Psi_{\text {FPS }}^{(n)}\left|\hat{P}_{\text {FPS }}\right| \Psi_{\text {FPS }}^{(n)}\right\rangle$}

Expanding the integral with the help of Eqs. (30) and (31) leads to

$$
\begin{aligned}
\left\langle\Psi_{\mathrm{FPS}}^{(n)}\left|\hat{P}_{\mathrm{FPS}}\right| \Psi_{\mathrm{FPS}}^{(n)}\right\rangle & =\left|\alpha_{q}^{(n)}\right|^{2}\left\langle\Psi_{q}^{B}\left|\hat{P}_{\mathrm{FPS}}\right| \Psi_{q}^{B}\right\rangle+\left|\alpha_{-q}^{(n)}\right|^{2}\left\langle\Psi_{-q}^{B}\left|\hat{P}_{\mathrm{FPS}}\right| \Psi_{-q}^{B}\right\rangle \\
& +\left(\alpha_{q}^{(n)} \alpha_{-q}^{(n) *} \int_{0}^{L} d x u_{q}^{2}(x) e^{i 2 q x}+\text { c.c. }\right) .
\end{aligned}
$$

Due to the periodicity of $u_{q}(x)$ and considering that $q_{j}^{\text {res }}=j \pi / n d$, the last integral vanishes:

$$
\begin{array}{r}
\int_{0}^{n d} d x u_{q}^{2}(x) e^{i 2 q x}=\left(\sum_{l=0}^{n-1} \exp (i 2 q l d)\right) \int_{0}^{d} u_{q}^{2}(x) e^{i 2 q x} d x \\
=\sum_{l=0}^{n-1}[\exp (i 2 \pi j / n)]^{l} \int_{0}^{d} u_{q}^{2}(x) e^{i 2 q x} d x=0,
\end{array}
$$

where the last identity is due to the vanishing of the geometric sum. Then together with the normalization (31), we end up with Eq. (37).

\section{APPENDIX C: SIMPLIFIED CALCULATION OF THE WAVEFUNCTION IN THE FPS}

As a supplementary result, once $\tilde{\alpha}_{-q}^{(n)}$ is calculated from (45), the values of $\Psi_{\mathrm{FPS}}^{(n)}(x)$ in one period, e.g. obtained with the transfer matrix approach, allow to calculate the periodic function $\tilde{u}_{q}(x)$. Solving (39a) for $\tilde{u}_{q}(x)$ we ob- tain:

$$
\tilde{u}_{q}(x)=\frac{\Psi_{\mathrm{FPS}}^{(n)}(x)-\tilde{\alpha}_{-q}^{(n)}\left(\Psi_{\mathrm{FPS}}^{(n)}\right)^{*}(x)}{1-\left|\tilde{\alpha}_{-q}^{(n)}\right|^{2}} \exp (-i q x) .
$$

Using (39a) and (45), the wavefunction in the entire FPS can be obtained easily.

\section{APPENDIX D: DERIVATION OF AN IDENTITY AND CALCULATION OF $\alpha_{q}^{(n)}$ AND $\alpha_{-q}^{(n)}$}

\section{Identity - first method}

From Eqs. (52), (35) and (23), i.e.

$$
\frac{L}{\tau_{\text {res }}^{(n)}}=v_{\text {res }}^{(n)}=\frac{\left\langle\Psi_{\mathrm{FPS}}^{(n)}\left|\hat{v} \hat{P}_{\mathrm{FPS}}\right| \Psi_{\mathrm{FPS}}^{(n)}\right\rangle}{\left\langle\Psi_{\mathrm{FPS}}^{(n)}\left|\hat{P}_{\mathrm{FPS}}\right| \Psi_{\mathrm{FPS}}^{(n)}\right\rangle},
$$

and Eqs. (15) and (7), i.e.

$$
\tau_{\text {res }}^{(n)}=\frac{1}{j_{i n}}\left\langle\Psi_{\mathrm{FPS}}^{(n)}\left|\hat{P}_{\mathrm{FPS}}\right| \Psi_{\mathrm{FPS}}^{(n)}\right\rangle,
$$

we derive the interesting identity, valid at resonance,

$$
L=\frac{1}{j_{i n}}\left\langle\Psi_{\mathrm{FPS}}^{(n)}\left|\hat{v} \hat{P}_{\mathrm{FPS}}\right| \Psi_{\mathrm{FPS}}^{(n)}\right\rangle,
$$


where the incoming probability current is given by $j_{i n}=$ $\hbar k / m$.

\section{Identity - second method}

This equation can also be derived directly from the conservation of electrical charge in the form

$$
\frac{\partial}{\partial t}|\Psi(x, t)|^{2}+\frac{\partial}{\partial x} j(x, t)=0,
$$

where $j$ is the one-dimensional probability current given by

$$
j(x, t)=\operatorname{Re}\left[\Psi^{*}\left(-\frac{i \hbar}{m} \frac{\partial}{\partial x}\right) \Psi\right] .
$$

Since in our case $|\Psi|^{2}$ and $j$ do not depend on time, Eq. (D4) simplifies to

$$
j(x, t)=\text { const. }
$$

Now we integrate the constant probability current density, Eq. (D5), first for the wave function to the left, i.e.
$\Psi_{L}(x)=\exp (i k x)$, and second for the wave function at resonance inside the FPS, i.e. $\Psi_{\mathrm{FPS}}^{(n)}(x)$, both over the intervall $[0, L]$. Then Eqs. (D6) and (34), yield also identity (D3).

\section{Calculation of $\alpha_{q}^{(n)}$ and $\alpha_{-q}^{(n)}$}

Making use of Eq. (36), (D3) and (39a) we can calculate $\alpha_{q}^{(n)}$ and $\alpha_{-q}^{(n)}$. Choosing the (arbitrary) phase of $\alpha_{q}^{(n)}$, so that $\alpha_{q}^{(n)}$ is real and positive, we obtain:

$$
\begin{aligned}
\alpha_{q}^{(n)} & =\left[\frac{2 \pi j_{i n}}{\left(1-\left|\tilde{\alpha}_{-q}^{(n)}\right|^{2}\right) v_{g}}\right]^{1 / 2}, \\
\alpha_{-q}^{(n)} & =\tilde{\alpha}_{-q}^{(n)} \alpha_{q}^{(n)},
\end{aligned}
$$

where $\tilde{\alpha}_{-q}^{(n)}$ is given by Eq. (45).
1 E. H. Hauge and J. A. Støvneng, Rev. Mod. Phys. 61, 917 (1989).

2 R. Landauer and T. Martin, Rev. Mod. Phys. 66, 217 (1994).

3 E. P. Wigner, Phys. Rev. 98, 145 (1955).

4 E. H. Hauge, J. P. Falck, and T. A. Fjeldly, Phys. Rev. B 36, 4203 (1987).

${ }^{5}$ C. R. Leavens and G. C. Aers, Phys. Rev. B 39, 1202 (1989).

6 M. S. Marinov and B. Segev, Phys. Rev. A 55, 3580 (1997).

7 D. Sokolovski and L. M. Baskin, Phys. Rev. A 36, 4604 (1987).

8 H. A. Fertig, Phys. Rev. Lett. 65, 2321 (1990).

9 D. Sokolovski and J. N. L. Connor, Phys. Rev. A 47, 4677 (1993).

10 M. Büttiker and R. Landauer, Phys. Rev. Lett. 49, 1739 (1982).

11 M. Büttiker, Phys. Rev. B 27, 6178 (1983).

12 J. P. Falck and E. H. Hauge, Phys. Rev. B 38, 3287 (1988).

13 C. R. Leavens and G. C. Aers, Phys. Rev. B 40, 5387 (1989).

14 T. Martin and R. Landauer, Phys. Rev. A 47, 2023 (1993).

15 V. Gasparian and M. Pollak, Phys. Rev. B 47, 2038 (1993).

16 V. Gasparian, M. Ortuño, J. Ruiz, E. Cuevas, and M. Pollak, Phys. Rev. B 51, 6743 (1995).

17 Z.-J. Li, J.-Q. Liang, and D. H. Kobe, Phys. Rev. A 65, 24101 (2002).

18 E. Pollak and W. H. Miller, Phys. Rev. Lett. 53, 115 (1984).

19 A. M. Steinberg, Phys. Rev. Lett. 74, 2405 (1995).

20 D. Sokolovski and J. N. L. Connor, Phys. Rev. A 42, 6512 (1990).

21 R. S. Dumont and T. L. Marchioro II, Phys. Rev. A 47,
85 (1993)

22 F. T. Smith, Phys. Rev. 118, 349 (1960).

23 W. Jaworski and D. M. Wardlaw, Phys. Rev. B 40, 6210 (1989).

24 P. Pereyra and E. Castillo, Phys. Rev. B 65, 205120 (2002).

25 C. Pacher and E. Gornik, Phys. Rev. B 68, 155319 (2003).

26 P. Pereyra, Phys. Rev. Lett. 84, 1772 (2000).

27 U. Merc, C. Pacher, M. Topic, F. Smole, and E. Gornik, Eur. Phys. J. B 35, 443 (2003).

28 To prevent some possible confusion we note that the transfer matrix can be also defined by $\left(\begin{array}{c}A_{R}^{+} \\ A_{R}^{-}\end{array}\right)=\hat{M}\left(\begin{array}{c}A_{L}^{+} \\ A_{L}^{-}\end{array}\right)$, for example used in Ref. 24. Of course $\hat{M}=M^{-1}$. The reader is asked to check carefully which definition is used when he consults the literature, since both occur frequently.

29 P. Erdös and R. C. Herndon, Adv. Phys. 31, 65 (1982).

${ }^{30}$ D. W. L. Sprung, H. Wu, and J. Martorell, Am. J. Phys. 61, 1118 (1993).

31 M. Born and E. Wolf, Principles of Optics (Dover Publ., Pergamon Press, Oxford, 1980), 6th ed.

32 D. J. Vezetti and M. Cahay, J. Phys. D 19, L53 (1986).

33 H. Yamamoto, Y. Kanie, and K. Taniguchi, phys. stat. sol. (b) 154, 195 (1989).

34 C. Pacher, C. Rauch, G. Strasser, E. Gornik, F. Elsholz, A. Wacker, G. Kießlich, and E. Schöll, Appl. Phys. Lett. 79, 1486 (2001).

35 F. Bloch, ZS. f. Phys. 52, 555 (1929).

36 There remains one special case when $t= \pm 1$. For $t= \pm 1$, this simple approach does not work because the resulting equations are then linear dependent. In that special case one would have to use explicitely the continuitiy of the probability current density. 
37 B. Ricco and M. Y. Azbel, Phys. Rev. B 29, 1970 (1980).

38 We note that in this case $|t|=1$, but $t \neq \pm 1$. Therefore it is no special case in the sense of footnote 36 and we can use the simple approach.

39 C. Rauch, G. Strasser, K. Unterrainer, W. Boxleitner,
E. Gornik, and A. Wacker, Phys. Rev. Lett. 81, 3495 (1998).

40 C. Kittel, Quantum Theory of Solids (John Wiley \& Sons, Inc., New York, 1987). 